\title{
Coronary Computed Tomography Angiography for the Diagnosis of Vasospastic Angina: Comparison with Invasive Coronary Angiography and Ergonovine Provocation Test
}

\author{
Jiesuck Park, $M D^{1}$, Hyung-Kwan Kim, MD, PhD ${ }^{1 *}$, Eun-Ah Park, MD, PhD ${ }^{2 *}$, Jun-Bean Park, MD, PhD ${ }^{1}$, \\ Seung-Pyo Lee, MD, PhD ${ }^{1}$, Whal Lee, MD, PhD², Yong-Jin Kim, MD, PhD ${ }^{1}$, Dae-Won Sohn, MD, PhD ${ }^{1}$ \\ ${ }^{1}$ Department of Internal Medicine and Cardiovascular Center, Seoul National University Hospital, Seoul, Korea; ${ }^{2}$ Department of Radiology, Seoul \\ National University Hospital, Seoul, Korea
}

Objective: To investigate the diagnostic validity of coronary computed tomography angiography (cCTA) in vasospastic angina (VA) and factors associated with discrepant results between invasive coronary angiography with the ergonovine provocation test (iCAG-EPT) and cCTA.

Materials and Methods: Of the 1397 patients diagnosed with VA from 2006 to 2016, 33 patients (75 lesions) with available cCTA data from within 6 months before iCAG-EPT were included. The severity of spasm (\% diameter stenosis [\%DS]) on iCAGEPT and CCTA was assessed, and the difference in \%DS $(\triangle \% \mathrm{DS})$ was calculated. $\triangle \% \mathrm{DS}$ was compared after classifying the lesions according to pre-cCTA-administered sublingual nitroglycerin (SL-NG) or beta-blockers. The lesions were further categorized with \%DS $\geq 50 \%$ on iCAG-EPT or CCTA defined as a significant spasm, and the diagnostic performance of cCTA on identifying significant spasm relative to iCAG-EPT was assessed.

Results: Compared to lesions without SL-NG treatment, those with SL-NG treatment showed a higher $\triangle \% \mathrm{DS}(39.2 \%$ vs. $22.1 \%$, $p=0.002)$. However, there was no difference in $\triangle \%$ DS with or without beta-blocker treatment $(35.1 \% \mathrm{vs} .32 .6 \%, p=0.643)$. The significant difference in $\triangle \% \mathrm{DS}$ associated with SL-NG was more prominent in patients who were aged $<60$ years, were male, had body mass index $<25 \mathrm{~kg} / \mathrm{m}^{2}$, and had no history of hypertension, diabetes, or dyslipidemia. Based on iCAG-EPT as the reference, the per-lesion-based sensitivity, specificity, positive predictive value, negative predictive value, and accuracy of CCTA for VA diagnosis were $7.5 \%, 94.0 \%, 60.0 \%, 47.1 \%$, and $48.0 \%$, respectively.

Conclusion: For patients with clinically suspected VA, confirmation with iCAG-EPT needs to be considered without completely excluding the diagnosis of VA simply based on CCTA results, although further prospective studies are required for confirmation. Keywords: Variant angina pectoris; Coronary computed tomography angiography; Coronary angiography; Provocation test; Nitroglycerin

\section{INTRODUCTION}

Vasospastic angina (VA) is characterized by acute chest pain at rest that is caused by contraction of the vascular smooth muscle tissue in large coronary arteries leading to coronary spasm $(1,2)$. According to the current

Received December 3, 2018; accepted after revision January 11, 2019.

*These authors contributed equally to this work.

Corresponding author: Hyung-Kwan Kim, MD, PhD, Department of Internal Medicine and Cardiovascular Center, Seoul National University Hospital, 101 Daehak-ro, Jongno-gu, Seoul 03080, Korea.

- Tel: (822) 2072-0243•Fax: (822) 762-9662•E-mail: cardiman73@gmail.com; and

Eun-Ah Park, MD, PhD, Department of Radiology, Seoul National University Hospital, 101 Daehak-ro, Jongno-gu, Seoul 03080, Korea. - Tel: (822) 2072-0187• Fax: (822) 747-7418•E-mail: iameuna1@gmail.com

This is an Open Access article distributed under the terms of the Creative Commons Attribution Non-Commercial License (https:// creativecommons.org/licenses/by-nc/4.0) which permits unrestricted non-commercial use, distribution, and reproduction in any medium, provided the original work is properly cited. 
guidelines, invasive coronary angiography (iCAG) with either an acetylcholine or ergonovine provocation test (EPT) is required for a conclusive diagnosis in patients with clinically suspected VA (1). However, the iCAG with provocation test is invasive and, thus, inherently poses some risks to the patient, such as myocardial ischemia or infarction, serious arrhythmia, and puncture-site related complications (3). The introduction of coronary computed tomography angiography (cCTA) has had a significant effect on clinical practice, especially in the diagnosis of coronary artery disease. Owing to its high sensitivity and negative predictive value (NPV), cCTA is now accepted as a powerful noninvasive tool not only for identifying anatomic features of coronary arteries (i.e., luminal stenosis or the presence of plaques), but also for predicting long-term outcomes (46). To accomplish this purpose, the acquisition of highquality images is critical, which can be realized by pre-cCTA administration of beta-blockers and sublingual nitroglycerin (SL-NG) for coronary artery dilation and heart rate reduction (7). Although cCTA has been widely used in the evaluation of patients with chest pain, there is a paucity of data regarding the impact of the use of beta-blocker and SL-NG on the diagnostic accuracy of CCTA in patients with suspected VA. Thus, the purpose of this study was to investigate the ability of cCTA to diagnose VA relative to the gold-standard iCAG-EPT. We also attempted to reveal the potential influence of pre-cCTA-administered beta-blockers and SL-NG on any discrepancy between iCAG-EPT and CCTA when assessing coronary vasospasm.

\section{MATERIALS AND METHODS}

\section{Study Population}

The selection process for the study population is presented in Figure 1. In brief, from January 2006 to December 2016, a total of 1397 patients who had a newly recorded diagnostic code of VA (I201) based on the International Classification of Disease, Tenth Revision, Clinical Modification were identified. Of them, 356 patients had undergone iCAG-EPT. Forty-nine of the 356 patients had undergone CCTA within 6 months before iCAG. Of them, patients with no anginal symptoms $(n=2)$ were

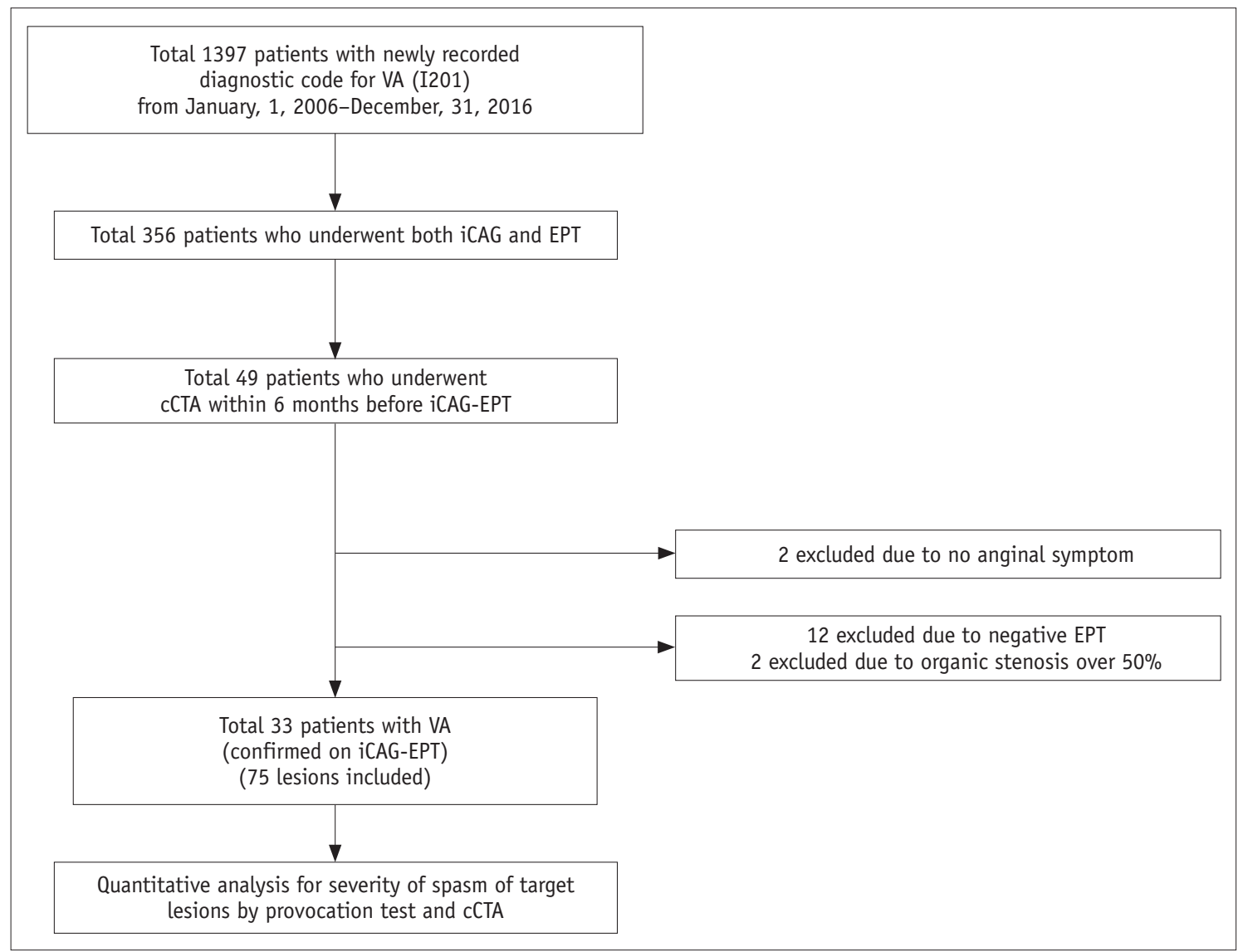

Fig. 1. Flow diagram showing study design and patient selection. $c C T A=$ coronary computed tomography angiography, EPT = ergonovine provocation test, $\mathrm{iCAG}=$ invasive coronary angiography, $\mathrm{VA}=$ vasospastic angina 
excluded. After iCAG-EPT, those with no spasm in any coronary trees upon the provocation test $(n=12)$ and with coronary spasm on lesions with significant stenosis (\% diameter stenosis $[\% D S] \geq 50 \%)(n=2)$ were further excluded. Finally, a total of 33 patients were included in the final analysis. Information regarding age; sex; body mass index (BMI); comorbidities including diabetes mellitus (DM), hypertension (HTN), dyslipidemia, and smoking; and administration of pre-cCTA medication including betablockers and SL-NG was collected by careful review of the electronic medical records. This study protocol was approved by the Institutional Review Board of Seoul National University Hospital and was conducted in accordance with the Declaration of Helsinki. The requirement for written informed consent was waived owing to the retrospective nature of the study.

\section{iCAG-EPT}

Experienced cardiologists performed iCAG via a transradial or transfemoral approach based on the current guidelines (8). Patients were instructed to stop using vasodilators, such as calcium channel blockers, nitrates, and potassium channel openers (i.e., nicorandil), 48 hours prior to the provocation test with the only exception of SLNG administration, if needed. First, diagnostic coronary angiograms for the left and right coronary arteries were obtained. Coronary lesions with an organic stenosis of $\% D S \geq 50 \%$ were excluded from the provocation test. The provocation test was then subsequently performed for the left and right coronary arteries using intracoronary ergonovine administration. Incremental doses of 30 (E1), 60 (E2), and $90 \mu \mathrm{g}$ (E3) were injected into the left coronary artery and incremental doses of 20 (E10), 40 (E2), and $60 \mu \mathrm{g}(\mathrm{E} 3)$ were injected into the right coronary artery (9). Care was taken to investigate both coronary arteries after the provocation test. After the provocation test was completed, intracoronary nitrate was injected to reverse coronary vasospasm.

\section{Multi-Detector CCTA Image Acquisition}

All CT examinations were performed using a dual-source CT scanner (SOMATOM Definition; Siemens Healthineers, Forchheim, Germany). Patients with a pre-scan heart rate of 65 beats per minute (bpm) or higher were given $50-100 \mathrm{mg}$ of oral metoprolol (Betaloc; AstraZeneca, Sweden) 45-60 minutes prior to the CT examination, unless the subject had a contraindication to beta-blockers. Additional beta- blockers were not administered to subjects who underwent CT scanning 45-60 minutes after administration of betablockers, even if the heart rate did not fall to below 65 bpm. SL-NG (0.6 mg; Nitroquick; Ethex, St. Louis, Mo, USA) was administered if the patient had no contraindications to nitroglycerin for coronary vasodilation immediately before scanning. Sixty $\mathrm{mL}$ of a nonionic contrast medium (iopromide, Ultravist ${ }^{\circledR}$ 370; Bayer Healthcare, Berlin, Germany) was injected into the antecubital vein at $5 \mathrm{~mL} /$ $\mathrm{sec}$, followed by an additional $20 \mathrm{~mL}$ of a nonionic contrast medium, and $50 \mathrm{~mL}$ of 8:2 mixture of normal saline and contrast medium, at a flow rate of $4 \mathrm{~mL} / \mathrm{sec}$ using a dual power injector (Stellant; Medrad, Indianola, PA, USA). The bolus triggering method was used to determine the starting point of $\mathrm{CT}$ acquisition by monitoring the signal density of contrast medium in the mid-ascending aorta. CT scans were initiated 8 seconds after a threshold trigger of 150 HU above baseline was achieved. Details of the CT scan protocol have been described in previous studies $(10,11)$.

\section{Image Interpretation of iCAG-EPT and CCTA}

All iCAG-EPT and CCTA images were carefully reviewed by two independent investigators who were blinded to patients' clinical information. All conflicting results were resolved by consensus $(\kappa=0.781)$. For target lesions on iCAG images, the degree of stenosis was evaluated using quantitative coronary analysis (QCA) (CAAS QCA; Esaote, Genova, Italy) at baseline and after the provocation test to calculate the $\%$ DS in the iCAG-EPT. QCA analysis was conducted with blinding to the clinical information and CCTA results.

The degree of stenosis on CCTA was assessed semiautomatically using a dedicated cardiac workstation (Syngo. via; Siemens Healthineers). Corresponding sites to each spastic lesion identified on iCAG-EPT were evaluated for severity of stenosis, presence of atherosclerotic plaques, and vascular remodeling. Because negative vascular remodeling was reported as a main finding of spasm on CCTA (12), its presence at the spasm sites was investigated. Negative remodeling was considered present when the vessel area at the site of plaque was smaller than the distal reference (12). Analysis of CCTA images was also performed blinded to the baseline clinical characteristics and results of iCAG-EPT.

\section{Statistical Analysis}

To evaluate the influence of pre-medication on discrepancies between the two modalities, the lesions were categorized into groups based on pre-test administration of 
beta-blockers and SL-NG, and the difference in \%DS ( $\triangle \% \mathrm{DS})$ of each lesion as measured iCAG-EPT and cCTA was compared between the groups. Subgroup analysis was performed to evaluate the impact of pre-medication on discrepancies in relation to accompanying cardiovascular risk factors such as old age (age $\geq 60$ years), obesity (BMI $\geq 25 \mathrm{~kg} / \mathrm{m}^{2}$ ), DM, HTN, dyslipidemia, and smoking. To determine whether temporal variance exists in diagnostic discrepancies, the lesions were further divided into groups according to when the CCTA was performed (from midnight to $10 \mathrm{AM}$ or $10 \mathrm{AM}$ to midnight) and compared for \%DS assessed by iCAG-EPT and cCTA.

To assess the diagnostic performance of cCTA compared to iCAG-EPT, lesions with \%DS more than 50\% after the provocation test or on CCTA were defined as a significant spasm (13). The lesions were then subsequently classified into four groups according to the spasm seen on iCAG-EPT and CCTA: 1) Matched-Insignificant: concordant insignificant spasm, 2) Matched-Significant: concordant significant spasm, 3) Mismatched-Significant: significant spasm only on iCAG-EPT, and 4) Mismatched-Insignificant: significant spasm only on CCTA without spasm on iCAG-EPT. To ensure matching of spasm sites on CCTA images with those on iCAG-EPT, great care was taken to identify the adjacent side branches and lumen ostia that were used as landmarks (12). The diagnostic performance of cCTA was determined using the sensitivity, specificity, positive predictive value (PPV), NPV, and accuracy compared with iCAG-EPT on both a perpatient and per-lesion basis.

All probability values were two-sided, and a $p$ value of $<$ 0.05 was considered statistically significant. The statistical package SPSS, version 22.0 (IBM Corp., Armonk, NY, USA) and $R$ version 3.3.3 (R Development Core Team, Vienna, Austria) were used for statistical analyses.

\section{RESULTS}

\section{Study Population}

Baseline characteristics of the study population are presented in Table 1. There were 27 men with a mean age of 59.3 years. The median time difference between CCTA and iCAG-EPT was 11 days. Of the 33 patients, nine underwent cCTA between midnight and 10 AM (21 lesions). Prior to CCTA, both beta-blocker and SL-NG were administered to 19 patients $(57.6 \%)$, beta-blocker alone to $5(15.2 \%)$, SL-NG alone to $6(18.2 \%)$, and neither beta-blocker nor SL-NG to 3 patients $(9.1 \%)$. Fifteen patients $(45.5 \%)$ had multi-vessel
Table 1. Baseline Characteristics of Study Population and Related Coronary Lesions

\begin{tabular}{|c|c|}
\hline \multicolumn{2}{|l|}{ Total patients $(n=33)$} \\
\hline \multicolumn{2}{|l|}{ Demographic information } \\
\hline Age, years & $59.3 \pm 13.9$ \\
\hline Male $(\%)$ & $27(81.8)$ \\
\hline BMI, $\mathrm{kg} / \mathrm{m}^{2}$ & $24.2 \pm 3.4$ \\
\hline \multicolumn{2}{|l|}{ Comorbidities (\%) } \\
\hline Hypertension & $17(51.5)$ \\
\hline Diabetes & $3(9.1)$ \\
\hline Dyslipidemia & $13(39.4)$ \\
\hline Smoking & $19(57.6)$ \\
\hline Alcohol consumption & $20(60.6)$ \\
\hline Time from cCTA to provocation test, days & $11(2-40)$ \\
\hline Time interval $\leq 30$ days $(\%)$ & $22(66.7)$ \\
\hline cCTA performed from midnight to $10 \mathrm{AM}(\%)$ & $9(27.3)$ \\
\hline Multi-vessel spasm (\%) & $15(45.5)$ \\
\hline \multicolumn{2}{|l|}{ Pre-test medication (\%) } \\
\hline No medication & $3(9.1)$ \\
\hline Beta-blocker only & $5(15.2)$ \\
\hline SL-NG only & $6(18.2)$ \\
\hline Beta-blocker and SL-NG & $19(57.6)$ \\
\hline \multicolumn{2}{|l|}{ Total lesions $(n=75)$} \\
\hline \multicolumn{2}{|l|}{ Lesion location (\%) } \\
\hline LAD & $24(35.8)$ \\
\hline LCX & $17(22.2)$ \\
\hline RCA & $34(42.0)$ \\
\hline Diffuse spasm & $34(45.3)$ \\
\hline \multicolumn{2}{|l|}{ Severity of stenosis } \\
\hline$\%$ DS by provocation test & $48.7 \pm 14.7$ \\
\hline$\%$ DS by cCTA & $14.4 \pm 17.2$ \\
\hline \multicolumn{2}{|l|}{ Visualized plaque in cCTA on per-lesion basis (\%) } \\
\hline Any plaque & $22(29.3)$ \\
\hline Non-calcified plaque & $7(9.3)$ \\
\hline Mixed plaque & $7(9.3)$ \\
\hline Calcified plaque & $8(10.7)$ \\
\hline Negative remodeling (\%) & $6(8.0)$ \\
\hline
\end{tabular}

Values given as mean \pm standard deviation, median (interquartile range, 25th and 75th percentile), or number (percentage). BMI = body mass index, cCTA = coronary computed tomography angiography, LAD = left anterior descending coronary artery, LCX $=$ left circumflex artery, RCA = right coronary artery, SL-NG = sublingual nitroglycerin, $\% \mathrm{DS}=\%$ diameter stenosis

coronary spasm. Of the 75 lesions identified using iCAGEPT, most were located in the right coronary artery (42.0\%) and $45.3 \%$ showed diffuse spasm. The mean \%DS recorded using iCAG-EPT and CCTA were $48.7 \pm 14.7$ and $14.4 \pm 17.2$, respectively. Plaque was visible in 22 lesions $(29.3 \%)$, and only $6(8.0 \%)$ lesions showed negative remodeling. 
Effect of cCTA Pre-Medication on the Discrepancies of Spasm Severity Assessed by iCAG-EPT and CCTA

In terms of SL-NG use, the $\triangle \% D S$ was significantly higher in lesions with pre-cCTA SL-NG $(39.2 \%$ vs. $22.1 \%, p=0.002)$

(Fig. 2). In contrast, there were no significant differences in $\triangle \% \mathrm{DS}$ according to the administration of pre-cCTA betablockers (35.1\% vs. $32.6 \%, p=0.643)$. Administration of SL-NG was associated with a significant difference in $\triangle \% \mathrm{DS}$ among those who were aged $<60$ years; were male; had BMI $<25 \mathrm{~kg} / \mathrm{m}^{2}$; and had no history of HTN, DM, or dyslipidemia, regardless of smoking history (Table 2). However, beta-blockers exerted no significant influence on discordance in $\triangle \% \mathrm{DS}$ regardless of the presence or absence of any cardiovascular risk factors. Although \%DS assessed using iCAG-EPT was higher compared to that obtained from CCTA regardless of when it was performed, there was no significant $\triangle \% \mathrm{DS}(50.5 \%$ vs. $48.3 \%, p=0.560$ by iCAGEPT and $14.0 \%$ vs. $15.0 \%, p=0.814$ by CCTA) and in $\triangle \%$ DS ( $36.6 \%$ vs. $33.2 \%, p=0.488$ ) between lesions from CCTA performed from midnight to $10 \mathrm{AM}$ or $10 \mathrm{AM}$ to midnight (Fig. 3).

\section{Diagnostic Performance of cCTA for Significant Spasms Compared to iCAG-EPT}

Of the 75 lesions, 40 (53.3\%) showed significant spasms on iCAG-EPT, whereas only five (6.7\%) did so on CCTA.

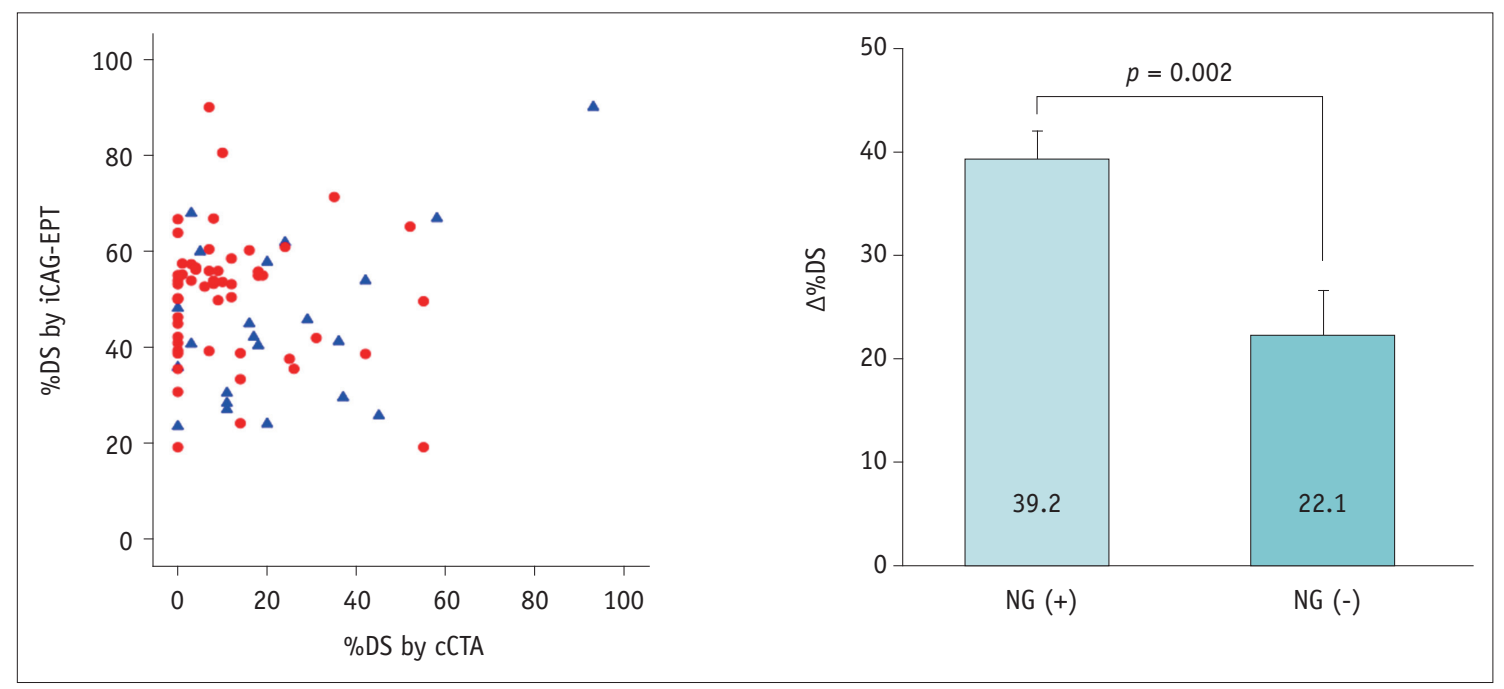

A

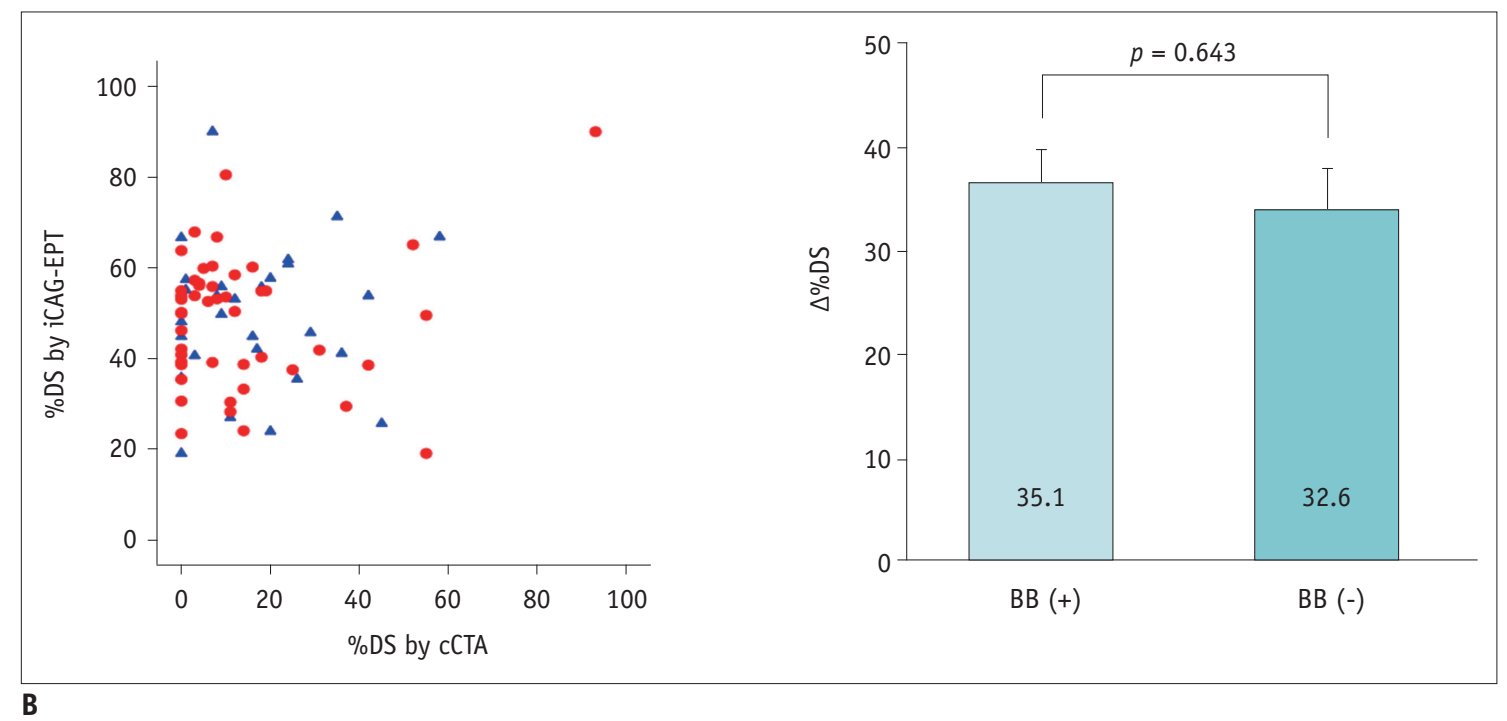

Fig. 2. Effect of pre-medication on difference in detecting severity of spasm between cCTA and iCAG-EPT.

A. SL-NG. B. BBs. BB = beta-blocker, iCAG-EPT $=$ iCAG with EPT, SL-NG $=$ sublingual nitroglycerine, $\% D S=\%$ diameter stenosis, $\triangle \% D S=$ difference in $\% \mathrm{DS}$ 
Table 2. Influence of cCTA Pre-Medication on Discrepancies of Spasm Severity Assessed by iCAG-EPT and cCTA According to Accompanied Cardiovascular Risk Factors

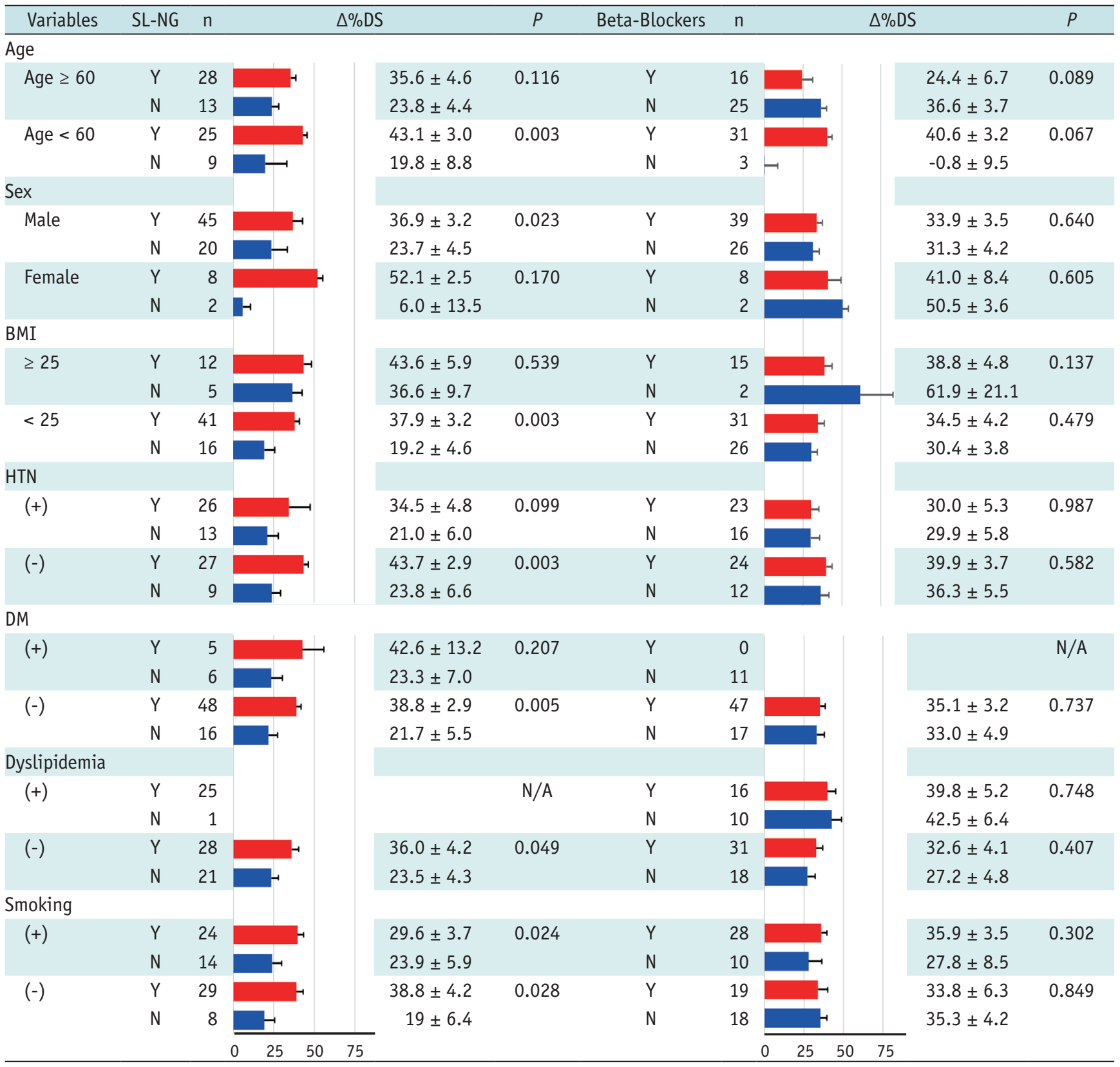

$\mathrm{DM}=$ diabetes mellitus, $\mathrm{HTN}=$ hypertension, $\mathrm{iCAG-EPT}=$ invasive coronary angiography with ergonovine provocation test, $\mathrm{N}=\mathrm{no}, \mathrm{N} / \mathrm{A}=$ not applicable, $\mathrm{Y}=$ yes, $\triangle \% \mathrm{DS}=$ difference in $\% \mathrm{DS}$

Overall, the discrepancy rate between the two diagnostic modalities was $52.0 \%$ (three $[4.0 \%$ ] Matched-Significant, 33 [44.0\%] Matched-Insignificant, 37 [49.3\%] MismatchedSignificant, and two [2.7\%] Mismatched-Insignificant lesions) (Figs. 4, 5). With iCAG-EPT as the reference standard, the sensitivity, specificity, PPV, NPV, and accuracy of CCTA for the diagnosis of VA were $7.5 \%, 94.0 \%$, $60.0 \%, 47.1 \%$, and $48.0 \%$, respectively on a per-lesion analysis, and $11.5 \%, 100.0 \%, 100.0 \%, 23.3 \%$, and $30.3 \%$, respectively on a per-patient analysis (Table 3 ).

\section{DISCUSSION}

The greatest clinical utility of CCTA is to reliably exclude fixed, obstructive coronary stenosis in patients with chest pain (5). Owing to its high NPV, cCTA was suggested to be a safe gatekeeper for iCAG $(14,15)$. Therefore, it is generally accepted that CCTA is the most accurate non- 


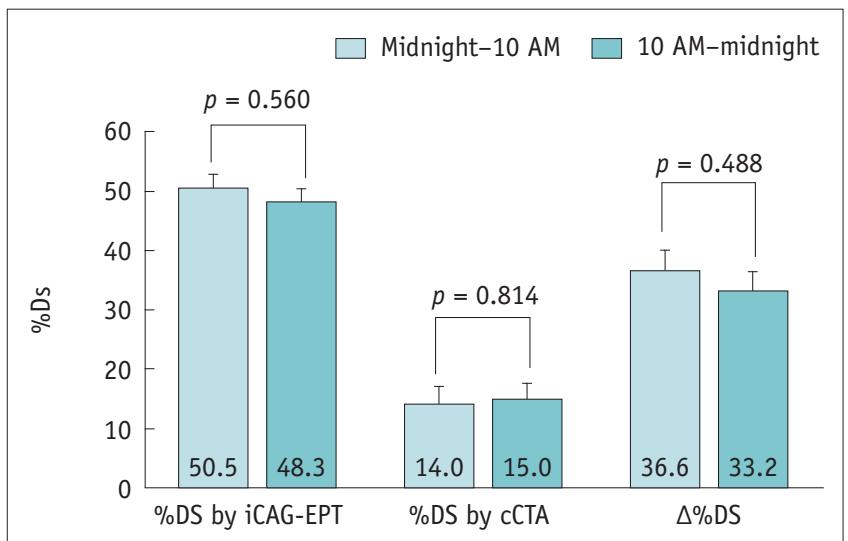

Fig. 3. Difference in severity of spasm between lesions according to time (from midnight to $10 \mathrm{AM}$ or $10 \mathrm{AM}$ to midnight) when CCTA was performed. invasive imaging tool for screening anatomically "fixed" coronary artery stenosis in patients with chest pain. Apart from obstructive and fixed coronary stenosis, coronary spasm also plays a pivotal role in creating acute chest pain. Coronary spasm as a predominant cause of acute chest pain is clinically referred to as VA, and this unique type of angina pectoris is frequently found in East Asian populations, including Korean $(1,2)$. According to guidelines (1), the confirmative diagnostic modality of choice for VA includes ICAG with ergonovine or acetylcholine provocation test, which can also provide anatomical information on the presence or absence of a high degree of fixed atherosclerotic stenosis. Further, as iCAG may induce rare but potentially life-threatening complications (3),
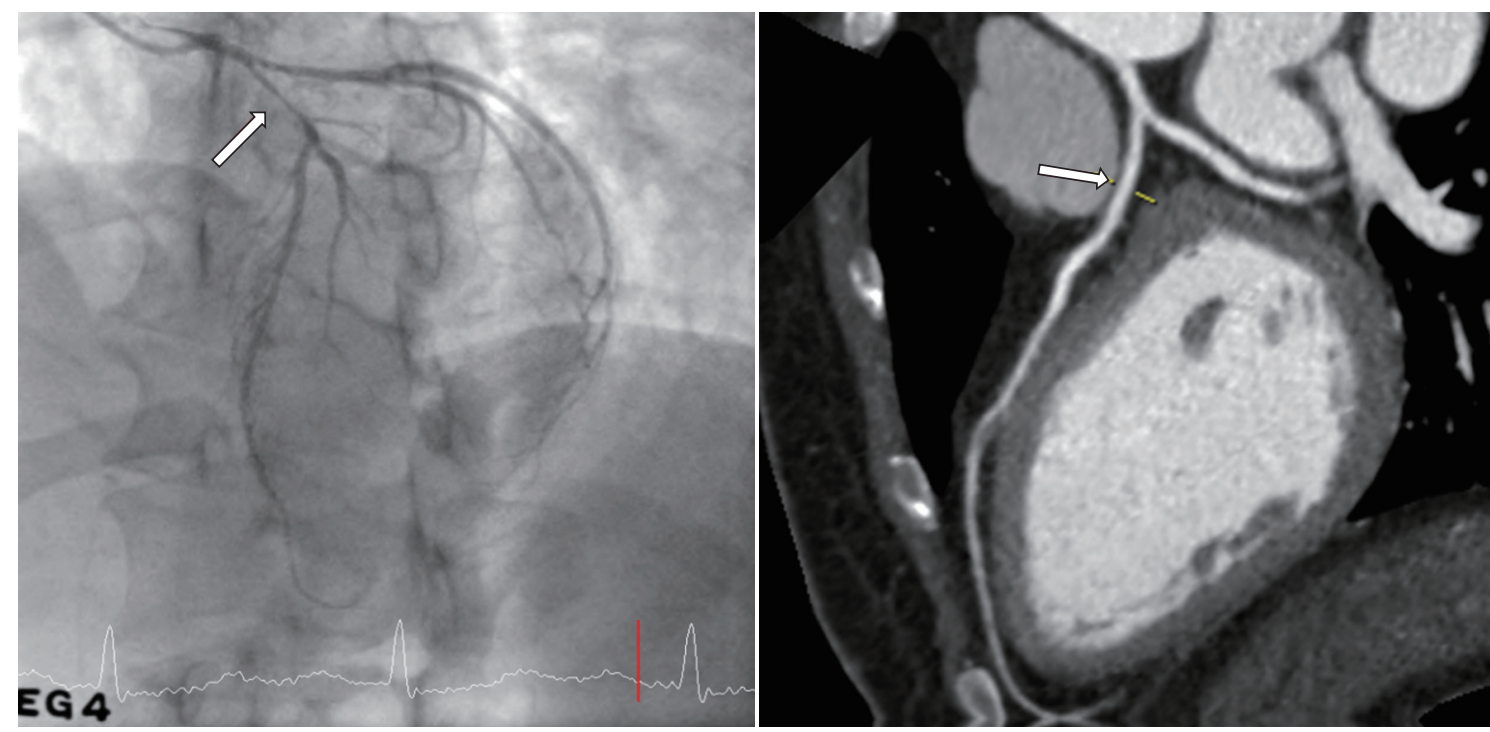

A
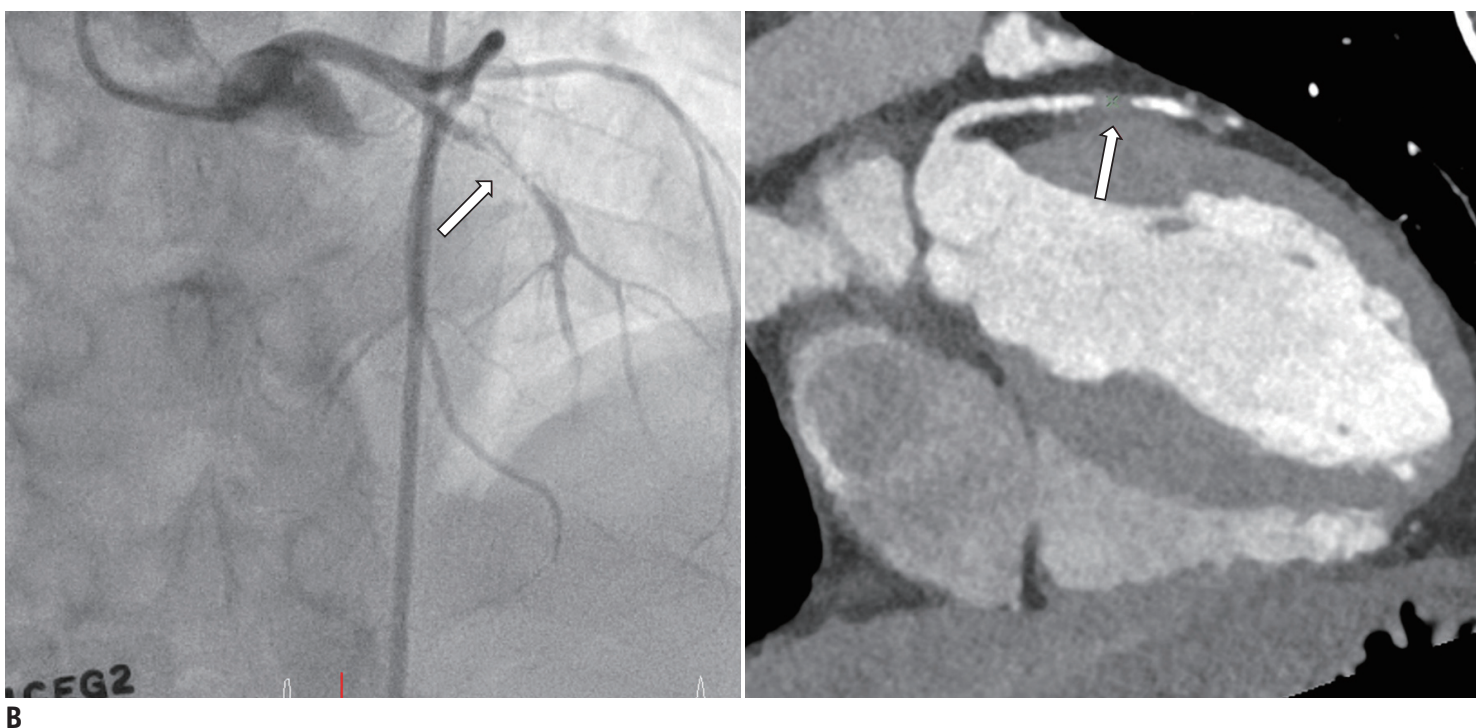

\section{B}

Fig. 4. Representative cases of Matched-Insignificant (A) and Matched-Significant (B) vasospasm between CCTA and iCAG-EPT. 
effort has been made to develop a non-invasive imaging modality for the diagnosis of VA, such as ergonovine stress echocardiography (16). However, ergonovine stress echocardiography requires a non-invasive treadmill test or nuclear myocardial scan to pre-exclude significant coronary stenosis (14). In contrast, given its high accuracy and NPV $(1,6,14)$, it has been suggested that cCTA would be an ideal imaging modality to simultaneously evaluate both anatomically fixed and vasospasm-induced stenosis. Several studies have suggested the possible role of cCTA as a gatekeeper to iCAG-EPT $(12,17-19)$. However, cases with false positive results of CCTA in VA have also been reported $(20,21)$, leaving the position of CCTA in the diagnosis or exclusion of VA in doubt. This may be attributed to differences in the characteristic findings in CCTA suggesting VA between studies and time-varying presentation of symptomatic vasospasm. To overcome this practical limitation, Kang et al. (17) and Jin et al. (19) proposed a new protocol dedicated to the diagnosis of VA. In brief,

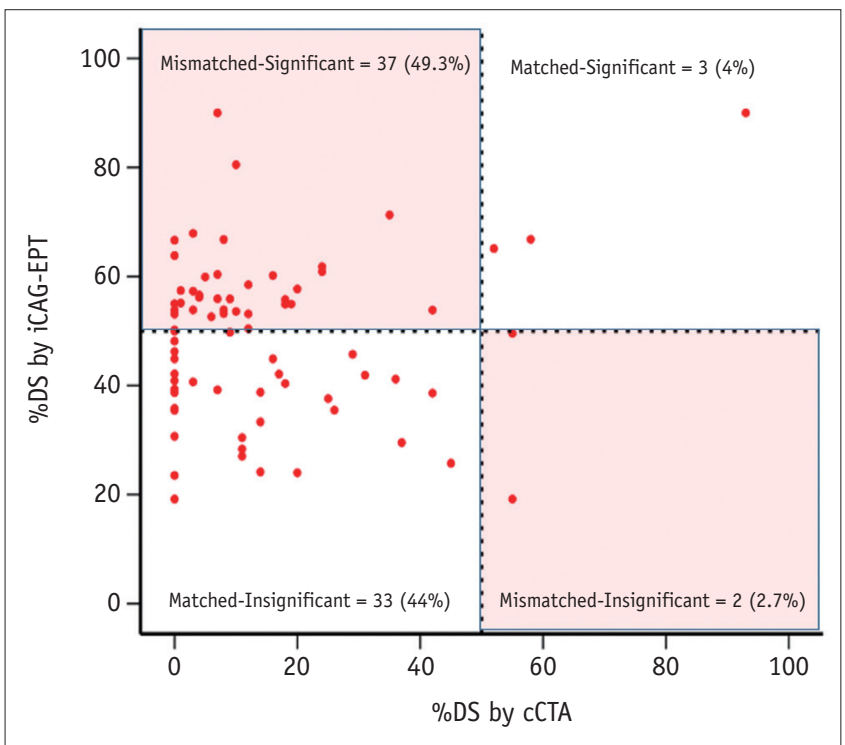

Fig. 5. Concordance or discordance between CCTA and iCAGEPT in differentiating significant from insignificant vasospasm with use of $50 \%$ cutoff value. twenty patents clinically suspected of having VA underwent baseline CCTA in the early morning without administration of a vasodilator before the test followed by repeated CCTA within 3 days with a continuous intravenous infusion of nitrate. With this new proposed protocol, they showed significant improvements in the diagnostic performance of cCTA for the detection of significant coronary vasospasm (73\%, 100\%, 100\%, and 56\% for the sensitivity, specificity, PPV, and NPV of CCTA in a per-patient-based analysis, respectively). Application of this new protocol, however, is practically limited because of the double administration of contrast agent, dual radiation exposure, and availability of CT scanners early in the morning. In contrast, the current study reports disappointing results; the concordance rate in the detection of significant vasospasm between iCAG-EPT and CCTA (i.e., true positive or true negative lesions) was found in only 36 out of 75 spastic lesions, resulting in low sensitivity of $7.5 \%$, NPV of $47.1 \%$, and accuracy of $48.0 \%$ on a per-lesion-based analysis. We also found that there was no significant difference in the severity of vasospasm on CCTA regardless of the time when the test was performed. Taking these results together, the approaches that omit vasodilator (i.e., SL-NG) administration prior to CCTA, especially in the early morning, could be more informative in diagnosing vasospasm. However, further studies with a large number of patients would be required to validate this suggested protocol for the diagnosis of vasospasm.

Previously, Ito et al. (12) found that atherosclerotic plaques, mostly those that were non-calcified, were observed at all spasm sites by CCTA. In the present study, however, we could not confirm their findings; $23(28.4 \%)$ lesions had atherosclerotic plaques and only seven lesions $(8.6 \%)$ involved non-calcified plaques, while most spasm sites were free of atherosclerotic plaques. It is not clear why the discrepancy arises, but we speculate that the discrepancy may be related to the spatial/temporal resolution of CT scanners (17). This speculation is based on a previous intravascular ultrasonographic study (a modality

Table 3. Diagnostic Performance of Coronary CT Angiography in Patients with Vasospastic Angina

\begin{tabular}{ccccccc}
\hline \multicolumn{2}{l}{ Per-Lesions Analysis Significant Spasm } & & & & \\
\hline iCAG & CCTA & Sensitivity & Specificity & PPV & NPV & Accuracy \\
$41(54.7 \%)$ & $5(6.67 \%)$ & $7.5 \%$ & $94.0 \%$ & $60.0 \%$ & $47.1 \%$ & $48.0 \%$ \\
\hline Per-Patient Analysis Significant Spasm & & & & & \\
\hline iCAG & CCTA & Sensitivity & Specificity & PPV & NPV & Accuracy \\
$26(78.8 \%)$ & $3(3.81 \%)$ & $11.5 \%$ & $100.0 \%$ & $100.0 \%$ & $23.3 \%$ & $30.3 \%$ \\
\hline
\end{tabular}

$\mathrm{NPV}=$ negative predictive value, $\mathrm{PPV}=$ positive predictive value 
with a high spatial/temporal resolution) showing the presence of non-calcified plaques at the spasm sites (22). In this respect, it is possible that further improvement in CCTA scanner technology with a higher spatial/temporal resolution may increase the diagnostic implication of non-calcified plaques at the spasm sites. To improve the diagnostic performance, previous studies $(17,18,22)$ combined various lesions characteristics including noncalcified plaques and negative vascular remodeling with significant stenosis, showing modest improvements in specificity and PPV. However, only six (8.0\%) of the spasm lesions showed signs of negative remodeling in the current study, suggesting a limited role for this finding in the detection of spasm sites.

Beta-blockers and SL-NG are routinely administered before acquisition of cCTA images to improve the quality of coronary artery images, if no contraindications exist (7). As both pre-medications can exert a significant influence on coronary vasoactivity, the diagnostic accuracy of cCTA can be affected by their use. One scenario that clinically matters is the situation where SL-NG administration leads to normal CCTA results in VA; based on negative CCTA results, timely referral of the patient to iCAG-EPT could be delayed, leading to delayed optimal treatment. In fact, $11(33.3 \%)$ patients in our series underwent iCAG-EPT more than 30 days after CCTA image acquisition. In addition, as beta-blockers can induce arterial spasm, their prescription in VA is discouraged among physicians. However, in the current study, we observed no significant association between pre-cCTA administration of beta-blockers and diagnostic discordance between CCTA and ICAG-EPT. This could be explained by the fact that beta-blockers, such as metoprolol, used for premedication mostly cause heart rate reduction via selective beta-1 receptor blockage without inducing coronary vasoconstriction mediated by beta-2 receptor blockage (23). Furthermore, regarding the impact of pre-cCTA medication according to the presence of coronary risk factors, SL-NG showed a consistent effect on discordance between iCAG-EPT and CCTA among patients who were aged $<60$ years; were male; had BMI $<25 \mathrm{~kg} / \mathrm{m}^{2}$; and had no history of HTN, DM, and dyslipidemia, which was not observed for beta-blocker usage. As endothelial dysfunction is known to be a major mechanism underlying coronary vasospasm $(24,25)$, those with a higher burden of cardiovascular risk factors may lead to endothelial dysfunction more severely, attenuating the effect of SL-NG on coronary vasodilation $(26,27)$.

Several limitations need to be acknowledged. First, this is a retrospective, observational study in a relatively small number of patients, and thus, a selection bias may be present. Therefore, further larger prospective studies need to be performed to consolidate the current findings. Second, the interval between CCTA and iCAG-EPT was over 1 month in 11 patients (33.3\%). Third, there might be a possibility of mismatch between the exact spasm sites on CCTA and those on iCAG-EPT. However, we tried our best to ensure matching of the spasm lesions on cCTA with iCAGEPT lesions by using side branches and ostia as landmarks. Fourth, as we analyzed only single CCTA results in patients with VA, temporal variation of vasospasm could not be analyzed in the current study. Lastly, although we used the EPT as a gold standard method to diagnose VA, a previous report showed a false negative result on iCAG-EPT (28).

In conclusion, for patients with clinically suspected VA, a final diagnosis made based on iCAG-EPT needs to be considered without excluding the diagnosis of VA simply based on insignificant results on CCTA, although further prospective studies in a large number of populations are required for confirmation. If confirmed, the development of a new CCTA protocol dedicated to the diagnosis of VA would be an interesting topic and of clinical importance.

\section{Conflicts of Interest}

The authors have no potential conflicts of interest to disclose.

ORCID iDs

Hyung-Kwan Kim

https://orcid.org/0000-0001-7950-2131

Eun-Ah Park

https://orcid.org/0000-0001-6203-1070

Jiesuck Park

https://orcid.org/0000-0001-6924-7106

\section{REFERENCES}

1. JCS Joint Working Group. Guidelines for diagnosis and treatment of patients with vasospastic angina (coronary spastic angina) (JCS 2013). Circ J 2014;78:2779-2801

2. Kim HL, Kim J, Kim HJ, Lim WH, Lee JY. Incidence and factors associated with mortality in 2,476 patients with variant angina in Korea. Sci Rep 2017;7:46031

3. Levenson B, Albrecht A, Göhring S, Haerer W, Reifart N, Ringwald G, et al. [6th report of the German Association of Cardiologists in private practice (BNK) on quality assurance in cardiac catheterization and coronary intervention 2006- 
2009]. Herz 2011;36:41-49

4. Cho I, Al'Aref SJ, Berger A, Ó Hartaigh B, Gransar H, Valenti $V$, et al. Prognostic value of coronary computed tomographic angiography findings in asymptomatic individuals: a 6-year follow-up from the prospective multicentre international CONFIRM study. Eur Heart J 2018;39:934-941

5. Raff GL, Chinnaiyan KM, Cury RC, Garcia MT, Hecht HS, Hollander JE, et al. SCCT guidelines on the use of coronary computed tomographic angiography for patients presenting with acute chest pain to the emergency department: a report of the Society of Cardiovascular Computed Tomography Guidelines Committee. J Cardiovasc Comput Tomogr 2014;8:254-271

6. Ferencik M, Mayrhofer T, Bittner D0, Emami H, Puchner SB, Lu MT, et al. Use of high-risk coronary atherosclerotic plaque detection for risk stratification of patients with stable chest pain: a secondary analysis of the promise randomized clinical trial. JAMA Cardiol 2018;3:144-152

7. Abbara S, Blanke P, Maroules CD, Cheezum M, Choi AD, Han $B K$, et al. SCCT guidelines for the performance and acquisition of coronary computed tomographic angiography: a report of the society of cardiovascular Computed Tomography Guidelines Committee: endorsed by the North American Society for Cardiovascular Imaging (NASCI). J Cardiovasc Comput Tomogr 2016;10:435-449

8. Levine GN, Bates ER, Blankenship JC, Bailey SR, Bittl JA, Cercek B, et al. 2011 ACCF/AHA/SCAI guideline for percutaneous coronary intervention. A report of the American College of Cardiology Foundation/American Heart Association task force on practice guidelines and the society for cardiovascular angiography and interventions. J Am Coll Cardiol 2011;58:e44-e122

9. Takagi Y, Yasuda S, Takahashi J, Tsunoda R, Ogata Y, Seki $A$, et al. Clinical implications of provocation tests for coronary artery spasm: safety, arrhythmic complications, and prognostic impact: multicentre registry study of the Japanese Coronary Spasm Association. Eur Heart J 2013;34:258-267

10. Moon JH, Park EA, Lee W, Yin YH, Chung JW, Park JH, et al. The diagnostic accuracy, image quality and radiation dose of 64-slice dual-source CT in daily practice: a single institution's experience. Korean J Radiol 2011;12:308-318

11. Park EA, Lee W, Kim KW, Kim KG, Thomas A, Chung JW, et al. Iterative reconstruction of dual-source coronary $\mathrm{CT}$ angiography: assessment of image quality and radiation dose. Int J Cardiovasc Imaging 2012;28:1775-1786

12. Ito T, Terashima M, Kaneda H, Nasu K, Ehara M, Kinoshita $Y$, et al. In vivo assessment of ergonovine-induced coronary artery spasm by 64 -slice multislice computed tomography. Circ Cardiovasc Imaging 2012;5:226-232

13. Shin DI, Baek SH, Her SH, Han SH, Ahn Y, Park KH, et al. The 24-month prognosis of patients with positive or intermediate results in the intracoronary ergonovine provocation test. JACC Cardiovasc Interv 2015;8:914-923

14. Dwivedi A, Al'Aref SJ, Lin FY, Min JK. Evaluation of atherosclerotic plaque in non-invasive coronary imaging. Korean Circ J 2018;48:124-133

15. Dewey M, Rief M, Martus P, Kendziora B, Feger S, Dreger H, et al. Evaluation of computed tomography in patients with atypical angina or chest pain clinically referred for invasive coronary angiography: randomised controlled trial. BMJ 2016;355:i5441

16. Song JK, Park SW, Kang DH, Hong MK, Kim JJ, Lee CW, et al. Safety and clinical impact of ergonovine stress echocardiography for diagnosis of coronary vasospasm. J Am Coll Cardiol 2000;35:1850-1856

17. Kang EJ, Kim MH, De Jin C, Seo J, Kim DW, Yoon SK, et al. Noninvasive detection of coronary vasospastic angina using a double-acquisition coronary CT angiography protocol in the presence and absence of an intravenous nitrate: a pilot study. Eur Radiol 2017;27:1136-1147

18. Kang KM, Choi SI, Chun EJ, Kim JA, Youn TJ, Choi DJ. Coronary vasospastic angina: assessment by multidetector CT coronary angiography. Korean J Radiol 2012;13:27-33

19. Jin CD, Kim MH, Kang EJ, Cho YR, Park TH, Lee KN, et al. Assessing vessel tone during coronary artery spasm by dualacquisition multidetector computed tomography angiography. Cardiology 2018;139:25-32

20. Ito K, Ogawa T, Yoshimura M. Severe coronary spasm occasionally detected by coronary computed tomography. Eur Heart J 2009;30:2768

21. Nakahara T, Toyama T, Tsushima Y, Kurabayashi M. Coronary vasospasm during CT angiography. J Cardiovasc Comput Tomogr 2014;8:328-330

22. Hong MK, Park SW, Lee CW, Ko JY, Kang DH, Song JK, et al. Intravascular ultrasound findings of negative arterial remodeling at sites of focal coronary spasm in patients with vasospastic angina. Am Heart J 2000;140:395-401

23. López-Sendón J, Swedberg K, McMurray J, Tamargo J, Maggioni AP, Dargie H, et al.; Task Force0n Beta-Blockers of the European Society of Cardiology. Expert consensus document on beta-adrenergic receptor blockers. Eur Heart J 2004; 25:1341-1362

24. Hadi HA, Suwaidi JA. Endothelial dysfunction in diabetes mellitus. Vasc Health Risk Manag 2007;3:853-876

25. Jansson PA. Endothelial dysfunction in insulin resistance and type 2 diabetes. J Intern Med 2007;262:173-183

26. Kugiyama K, Ohgushi M, Motoyama T, Sugiyama S, Ogawa H, Yoshimura M, et al. Nitric oxide-mediated flow-dependent dilation is impaired in coronary arteries in patients with coronary spastic angina. J Am Coll Cardiol 1997;30:920-926

27. Kugiyama K, Yasue H, Okumura K, Ogawa H, Fujimoto K, Nakao K, et al. Nitric oxide activity is deficient in spasm arteries of patients with coronary spastic angina. Circulation 1996;94:266-271

28. Yasue H, Nakagawa H, Itoh T, Harada E, Mizuno Y. Coronary artery spasm--clinical features, diagnosis, pathogenesis, and treatment. J Cardiol 2008;51:2-17 